\title{
Primary care consultation behaviours of long-term, adult survivors of cancer in the UK
}

\author{
Nada F Khan, Eila Watson and Peter W Rose
}

\author{
ABSTRACT \\ The population of cancer survivors is growing, and GPs \\ have an increasing role in their care. The General \\ Practice Research Database was used to compare \\ consultation rates between cancer survivors and \\ controls. Breast and colorectal cancer survivors had \\ one more consultation per year compared with controls \\ up to 5 years after diagnosis; rates then converged at \\ 10 years post-diagnosis. Prostate cancer survivors \\ consistently consulted up to three more times per year \\ than controls. These increased consultation rates are \\ leading to an impact on service capacity. \\ Keywords \\ family practice; long-term care; neoplasms; primary \\ health care; survivors.
}

\section{INTRODUCTION}

The prevalence of cancer survivors has increased due to demographic changes, increasing incidence of cancer, and improvements in cancer diagnosis and treatment. Current estimates indicate that 2 million people in the UK are cancer survivors; and at the current rate of growth, there will be 4 million cancer survivors in the UK by $2030 .^{1}$ GPs are responsible for the majority of cancer-related and comprehensive healthcare needs in this population after discharge from hospital follow-up. However, little is known about how cancer survivors use primary care services. The aims of this research are to describe the consultation patterns of adult survivors of breast, colorectal, and prostate cancer in British primary care at different points of survival, and to explore whether there are differences in the consultation rates between cancer survivors and a matched control population.

\section{METHOD}

This analysis uses the UK General Practice Research Database (GPRD). Cancer survivors were defined as those aged 30 years or over at the time of diagnosis of breast, colorectal, or prostate cancer, with at least 5 years of survival postdiagnosis. Controls were matched on the basis of age (within 1 year), sex, and primary care practice, and the analysis was undertaken for a time period from 1 September 2003 to 31 August 2006. A more detailed description of the data source and patient inclusion and exclusion criteria has been reported previously. ${ }^{2}$ Descriptive statistics were used to describe consultation rates according to length of survival, and regression analysis to compare the number of consultations between survivors and controls matched by age, sex, and primary care practice. All analyses were performed using Stata MP (version 10.1).

First, the total number of consultations for each patient over the analysis period was counted. Follow-up was censored upon death or transfer out of the GPRD primary care practice; therefore, the consultation rate was standardised to the number 


\section{How this fits in}

Little is known about the primary care consultation patterns of the increasing population of cancer survivors in the UK. This study shows that breast and colorectal cancer survivors have higher rates of GP consultations in the first 10 years post-diagnosis, while prostate cancer survivor consultation rates are elevated past this point. of years each patient contributed data to the followup. Face-to-face consultations in the practice with a member of the primary care team and home visits were counted, excluding events such as patient correspondence, claim payments, and other administrative events. The 'stsplit' command in Stata was used to account for the fact that patients moved through survival periods during the 3-year period; for instance, a person diagnosed with cancer in 1998 was counted as a 5-year survivor in 2003 and a 6-year survivor in 2004.

To compare the total number of consultations between cancer survivors and matched controls over the analysis period, multivariate fixed-effects negative binomial regression was used. ${ }^{3}$ Differences in the number of consultations are expressed as incidence rate ratios (IRRs). The analysis adjusted for comorbidity using an adaptation of the Charlson comorbidity index. ${ }^{4}$

\section{RESULTS}

The dataset contains longitudinal primary care data on 18612 breast, 5764 colorectal, and 4868 prostate cancer survivors, and 116418 control patients. The cohort had a median age of 72 years in 2003 (interquartile range 61 to 80 years). Just over half $(52 \%)$ of the cancer survivors had a Charlson score greater than zero, indicating at least one additional comorbidity in their electronic medical record.

Descriptive results show that breast and colorectal cancer survivors have a significantly increased consultation rate up to 10 years from diagnosis, with one extra consultation per year up to 5 years for breast cancer and up to 9 years for colorectal cancer (Figures 1 and 2). The multivariate, matched analysis showed a slight increase in the overall number of consultations between breast cancer survivors (IRR 1.11, 99\% confidence interval $[\mathrm{Cl}]=1.09$ to 1.12 ) and colorectal survivors (IRR $1.12,99 \% \mathrm{Cl}=1.08$ to 1.14 ) versus controls. In comparison, prostate cancer survivors consulted up to three more times annually compared to controls, and this trend persisted even 15 years postdiagnosis (Figure 3). Compared to matched controls, prostate cancer survivors had $39 \%$ more consultations over the 3-year follow-up period (IRR $1.39,99 \% \mathrm{Cl}=1.35$ to 1.43 ).

It is possible that patients who died contributed disproportionately to the excess consultation rate. However, after excluding patients who died during the analysis period, there was still an increase in consultations among breast (IRR 1.07, 95\% Cl = 1.06 to 1.09 ), colorectal (IRR $1.08,95 \% \mathrm{Cl}=1.05$ to 1.11), and prostate (IRR $1.33,95 \% \mathrm{Cl}=1.29$ to 1.37) cancer survivors compared to controls. 


\section{DISCUSSION}

\section{Summary of main findings}

The analysis of consultation rates among cancer survivors in the GPRD has shown a significant difference in the number of consultations between survivors and matched controls, with the greatest difference for prostate cancer survivors. Breast and colorectal cancer survivors have higher consultation rates than a control population, until rates converge 10 years post-diagnosis. The increase in consultation rates for prostate cancer survivors persists for many years post-diagnosis.

A major contribution to the increased consultation rate in prostate cancer will be the monitoring and administration of hormonal treatments; this will apply to breast cancer patients as well, but to a lesser extent. Half of cancer survivors have unmet needs relating to ongoing health conditions, which include secondary effects from the disease and its treatment, risk of second cancers, and emotional responses such as depression and anxiety. ${ }^{5-6}$ The early peak in consultation rates among all groups of survivors may reflect the need to address in primary care aspects of ongoing morbidity related to the cancer.

\section{Strengths and limitations of the study}

This is the first study of primary care consulting behaviours among cancer survivors in the UK. The study used data from a large, representative database, and explored consultation behaviour among a community-based group of cancer survivors, with a robust comparison population. It was not possible to confirm the reasons for increased consultation rates, due to the multitude of possible reasons for GP visits, and further research could clarify this point.

\section{Comparison with existing literature}

Surveys of long-term cancer survivors in Norway and France have indicated an increase in the use of GP services compared to a control population, but there is little previous research looking at the primary care consultation patterns of survivors of cancer in the UK. ${ }^{7-8}$ Future research should consider reasons for increased health services use in this growing population.

\section{Implications for future research and clinical practice}

These trends in increased consultation behaviours are impacting on the workload in British primary care, and this impact will increase as the population of cancer survivors continues to grow. As many as one in six adults over the age of 65 years seen by a GP is likely to be a cancer survivor, and a significant proportion of these will be breast, colorectal, or prostate cancer survivors. ${ }^{9}$ It is estimated that an average GP with a list size of 2000 patients would provide 60-180 extra consultations per year for all cancer patients, assuming a cancer prevalence of $3 \%$ in the UK, and the extra consultation rate for all cancers mirrors the one to three extra consultations per year of the three cancers in this study. ${ }^{1}$ Additionally, for some cancers, such as breast, colorectal, and prostate, the current trend is towards earlier discharge to primary care. Many of these long-term cancer survivors will not require specialist disease management, and this paper quantifies the likely effect on GP workload.

\section{Funding body}

This work has been funded by Macmillan Cancer Support through its Research Capacity Development Programme and by Cancer Research UK (CR-UK) grant number C23140/A8854

\section{Ethical approval}

The GPRD Group has obtained ethical approval for all observational research using GPRD data. This study has been approved by the Independent Scientific Advisory Committee (ISAC) of the GPRD (ISAC protocol No. 06_051R).

\section{Competing interests}

The authors have stated that there are none.

\section{Discuss this article}

Contribute and read comments about this article on the Discussion Forum: http://www.rcgp.org.uk/bjgp-discuss

\section{REFERENCES}

1. Maddams J, Brewster D, Gavin A, et al. Cancer prevalence in the United Kingdom: estimates for 2008. Br J Cancer 2009; 101(3): 541-547.

2. Khan NF, Carpenter L, Watson E, Rose PW. Cancer screening and preventative care among long-term cancer survivors in the United Kingdom. Br J Cancer 2010; 102(7): 1085-1090.

3. Rabe-Hesketh S, Skrondal A. Multilevel and longitudinal modeling using Stata. 2nd edn. College Station, Texas: Stata Press, 2008.

4. Khan NF, Perera R, Harper S, Rose PW. Adaptation and validation of the Charlson Index for Read/OXMIS coded databases. BMC Fam Pract 2010; 11: 1

5. Cancer survivors: living longer, and now, better. Lancet 2004; 364(9452): 2153-2154.

6. Armes J, Crowe M, Colbourne L, et al. Patients's supportive care needs beyond the end of cancer treatment: a prospective, longitudinal survey. J Clin Oncol 2009; 27(36): 6172-6179.

7. Nord, C, Mykletun A, Thorsen L, et al. Self-reported health and use of health care services in long-term cancer survivors. Int J Cancer 2005; 114: 307-316.

8. Joly F, HenryAmar M, Arveux P, et al. Late psychosocial sequelae in Hodgkin's disease survivors: a French population based case-control study. J Clin Oncol 1996; 14: 2444-2453.

9. Grunfeld E. Cancer survivorship: a challenge for primary care physicians. Br J Gen Pract 2005; 55(519): 741-742. 